\title{
Die mit den Füßen
}

\author{
Aus einer Fußmassage hat sie eine komplexe Behandlungs- \\ methode entwickelt, mit Neugierde, Gespür für Menschen, \\ differenzierter Beobachtung und Intuition. DHZ-Redakteur \\ Christian Böser zu Besuch bei HANNE MARQUARDT.
}
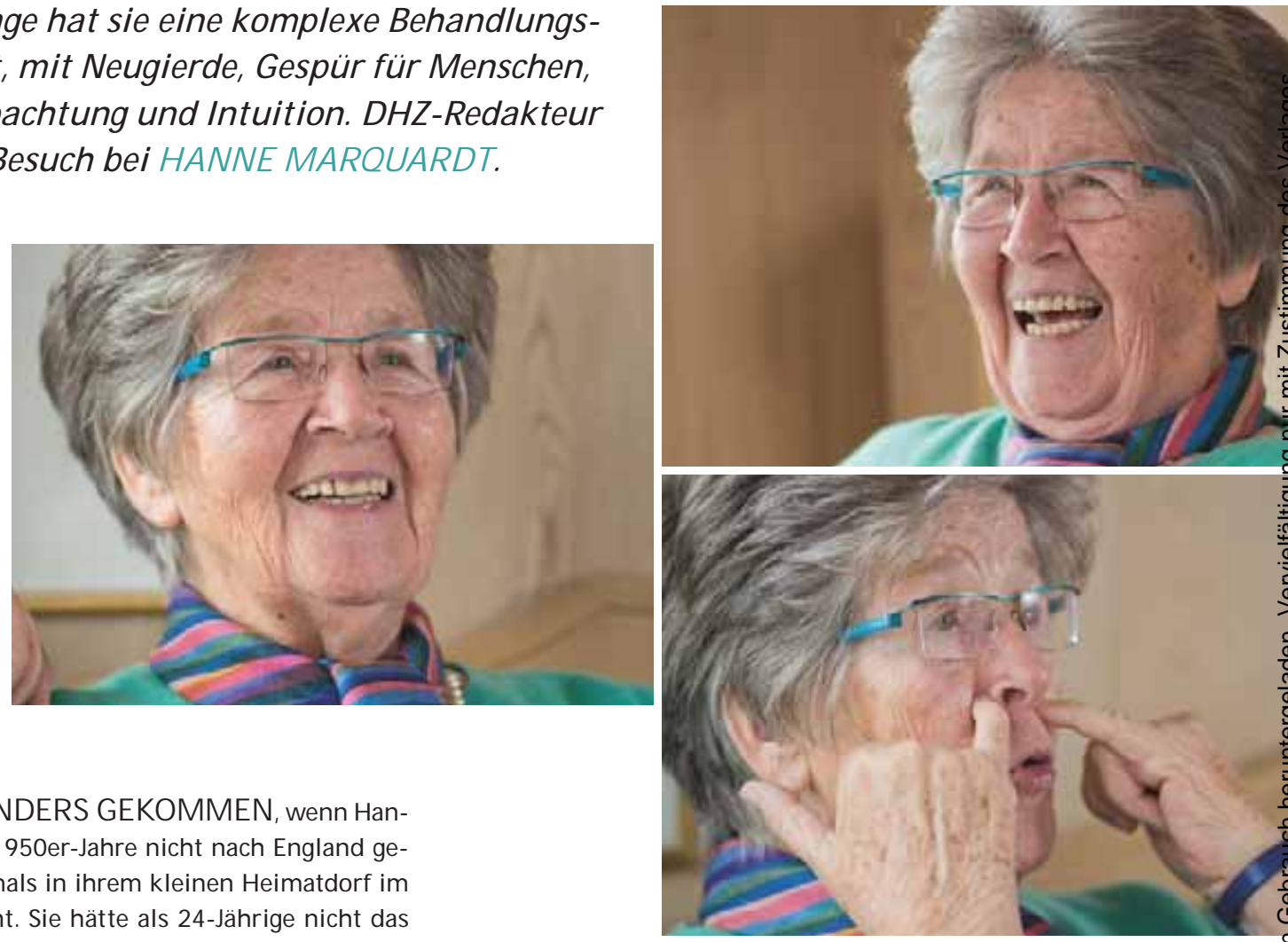

AUESWÄRE GANZ ANDERS GEKOMMEN, wenn Hanne Marquardt Anfang der 1950er-Jahre nicht nach England gegangen wäre. Sie hätte damals in ihrem kleinen Heimatdorf im Allgäu kein Englisch gelernt. Sie hätte als 24-Jährige nicht das Büchlein „Stories the feet can tell“ lesen können, verfasst von der Amerikanerin Eunice Ingham. Sie hätte nicht die von Ingham entwickelte Fußmassage zur Gesunderhaltung kennengelernt. Sie hätte daraus nicht die Reflexzonentherapie am Fuß entwickeln können. „Vieles in meinem Leben hat sich einfach zum rechten Zeitpunkt ergeben“, sagt Hanne Marquardt. Ihr Leben überhaupt sei eine „Reihung guter Fügungen.“

Hanne Marquardt, 81 Jahre alt, sitzt an diesem grauen Donnerstag im November vergangenen Jahres gemütlich auf ihrem blauen Sofa in Burgberg im Schwarzwald. Sie trägt eine hellblaue Jeans, trotz der ersten Schneeflocken in diesem Jahr einen kurzärmligen grünen Pullover, ein buntes Tuch und eine Perlenkette um den Hals. Sie ist eine zuvorkommende Gastgeberin, schenkt Kräutertee aus eigenem Anbau ein, bietet „Käsfüße“ an, ein mit Käse überzogenes Gebäck in Form eines Fußes. Und ganz nebenbei beginnt sie auch schon, noch bevor die erste Frage gestellt ist, von ihrem „Fuß-Weg“ zu erzählen, wie sie es nennt. „Sie können mich gern unterbrechen ", sagt sie und wird diesen Satz noch zweimal in den folgenden beiden Stunden wiederholen. Doch Unterbrechungen werden nicht nötig sein, denn Hanne Marquardt erzählt fesselnd, was ihr Besucher erfahren möchte: Wie aus der präventiven Fußmassage eine komplexe Behandlungsmethode wurde, welche Rolle dabei die "guten Fügungen“ gespielt haben und was es mit den „vielen eigenen Erfahrungen und daraus gezogenen praktischen Konsequenzen“ auf sich hat, wie sie es nennt.

\section{Erste Schritte}

Nach ihrem Schulabschluss geht Hanne Marquardt nach England und lässt sich dort zur Krankenschwester ausbilden. Sie ist Anfang 20, als sie mit dem Examen in der Tasche nach Deutschland zurückkehrt. Beruflich angekommen fühlt sie sich damals aber noch nicht. Weil sie gerne mit Menschen arbeitet, und gerne auch mit den Händen, beschließt sie, eine weitere Ausbildung zu absolvieren: Sie wird Masseurin. Wie bedeutend dieser Schritt für ihren weiteren Lebensweg ist, wird ihr erst im Nachhinein klar werden.

Einige Jahre später nimmt sie eine Stelle in einer Kurklinik in Mühringen bei Horb am Neckar an. Dort massiert sie eines Tages eine amerikanische Patientin, und die bittet sie, ihr auch die Füße intensiv „durchzukneten“, das tue ihr besonders gut. Die Amerikanerin zeigt Hanne Marquardt dafür einige einfache Griffe. Ohne es zu wissen, kommt sie dabei erstmals mit der reflexology in Berührung. „Ich wusste nicht, dass man Füße auch auf eine ganz andere, spezielle Art behandeln kann. “ Die Füße gehören fortan mit dazu, wenn die Patientin zur Massage kommt.

Am Ende ihrer Kur schenkt die Amerikanerin Hanne Marquardt das Büchlein von Eunice Ingham, das ihr Leben nachhaltig verändern wird. Sie liest darin von der reflexology und den erstaunlichen Behandlungsergebnissen. Doch sie ist zunächst skep- 
Ich mag den Begriff „Heilerin“ nicht, denn ich hatte noch nie den Eindruck, dass ich einen Menschen heilen konnte. Meine Aufgabe ist lediglich, die Selbstheilungskräfte im Patienten anzuregen und gut zu unterstützen.

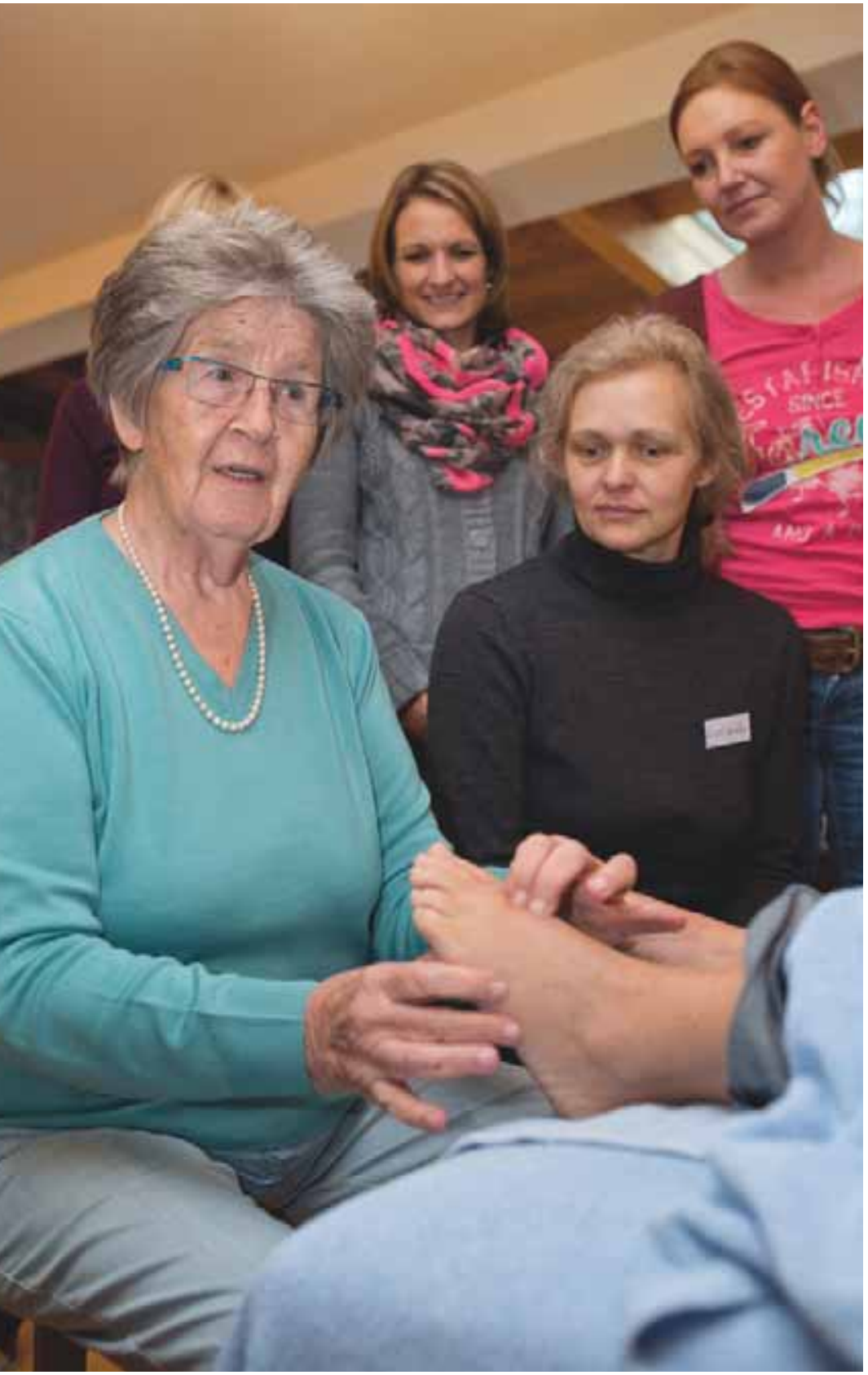

ganz offensichtlich gutgetan, seine kalten Füße wurden warm.“ Nach der Behandlung verlässt der Patient zufrieden die Praxis. Doch am nächsten Morgen um 7:00 Uhr ruft er an: Sein Bett habe in der Nacht dreimal frisch überzogen werden müssen, Darm und Blase hätten „überdeutlich reagiert.“ Er habe abwechselnd Hitzeschübe und Schüttelfrost bekommen, sei teilweise aggressiv und depressiv gewesen und hätte keine Ruhe gefunden, erzählt Hanne Marquardt von dem Telefonat und lacht dabei über sich sel bst. „Ich habe inm zerknirscht Abbitte getan und dachte, das war's wohl.“ Zu ihrer Überraschung bittet der Patient jedoch gleich um einen neuen Termin. „Als ich ihn das nächste Mal behandelt habe, habe ich überlegt, an welchen Zeichen ich die Grenze seiner Belastbarkeit erkennen könnte." Als er bei einem bestimmten Griff sagt, dass seine Hände ganz plötzlich feucht werden, erkennt sie, dass sein vegetatives Nervensystem stark reagiert.

Es sind Erlebnisse wie dieses, die Hanne Marquardt nach und nach weitere Hinweise auf die Dosierungsgrenzen geben, zusätzlich zum starken Schmerz an belasteten Zonen, der bis dahin als das einzige Signal gilt. Zu feuchten Händen und Füßen kommen trockener Mund, veränderte Gesichtsfarbe, erhöhte Pulsfrequenz, Veränderung der Körpertemperatur und vieles mehr hinzu.

\section{Die Bew usstwerdung}

Hanne Marquardt steht von ihrem Sofa auf, holt ihre Broschüre „Formähnlichkeiten als Schlüssel zur Therapie“ aus ihrem Bücherregal und zeigt dem Besucher das Bild eines Fußes. „In den bekannten und den neu gefundenen Zonen habe ich nach und nach immer deutlicher das Prinzip der Formenähnlichkeit zwischen einem aufrecht stehenden Fuß und einem sitzenden Menschen erkannt. Das lieferte mir einen verlässlichen Schlüssel zum genaueren Auffinden der einzelnen Reflexzonen. Eine Form hat immer eine Information, und sie dient zugleich einer Funktion. Sie ist das Ergebnis eines vorherigen feinstofflichen Prozesses, der sich allmählich zu Materie verdichtet. "Einfach ist es nicht, dieser Erklärung zu folgen. Doch dann spricht sie von Paracelsus, der im Mittelalter bei Heilpflanzen ein ähnliches Prinzip entdeckt hatte, dieSignaturenlehre, und von der Ohrakupunktur nach Nogier, die dieses Schema bestätigt: Die Form der Ohrmuschel, sie zeigt eine offensichtliche Ähnlichkeit mit der eines Embryos. Jetzt ist das Prinzip klarer.

Während der Entwicklung der RZF ergab es sich von selbst, dass Hanne Marquardt Brücken geschlagen hat zu anderen bekannten Naturheilverfahren. „Ich habe mir Anregungen geholt bei anderen Experten und sie in meine Arbeit integriert." Sie entdeckt die energetischen Zusammenhänge zwischen den Zähnen und den Füßen, sowie eine Möglichkeit der nicht invasiven Narbenbehandlung über die Reflexzonen. Sie verwendet regulierende Griffe aus der Eutonie, entwickelt die Lymphbehandlung an den Fußzonen, findet Meridianzusammenhänge und vieles mehr.

\section{Ein heilsamer Schock}

An die erste Behandlung an ihren eigenen Füßen kann sich Hanne Marquardt noch gut erinnern. „Es war wie ein heilsamer Schock: Ich habe zum ersten Mal sel ber gespürt, wie schmerzhaft eine belastete Reflexzone sein kann.“ Dabei bekam sie die erste Fußreflexzonenmassage von Eunice Ingham persönlich. Neun Jahre nachdem sie das Buch über die reflexology erstmals gele- 


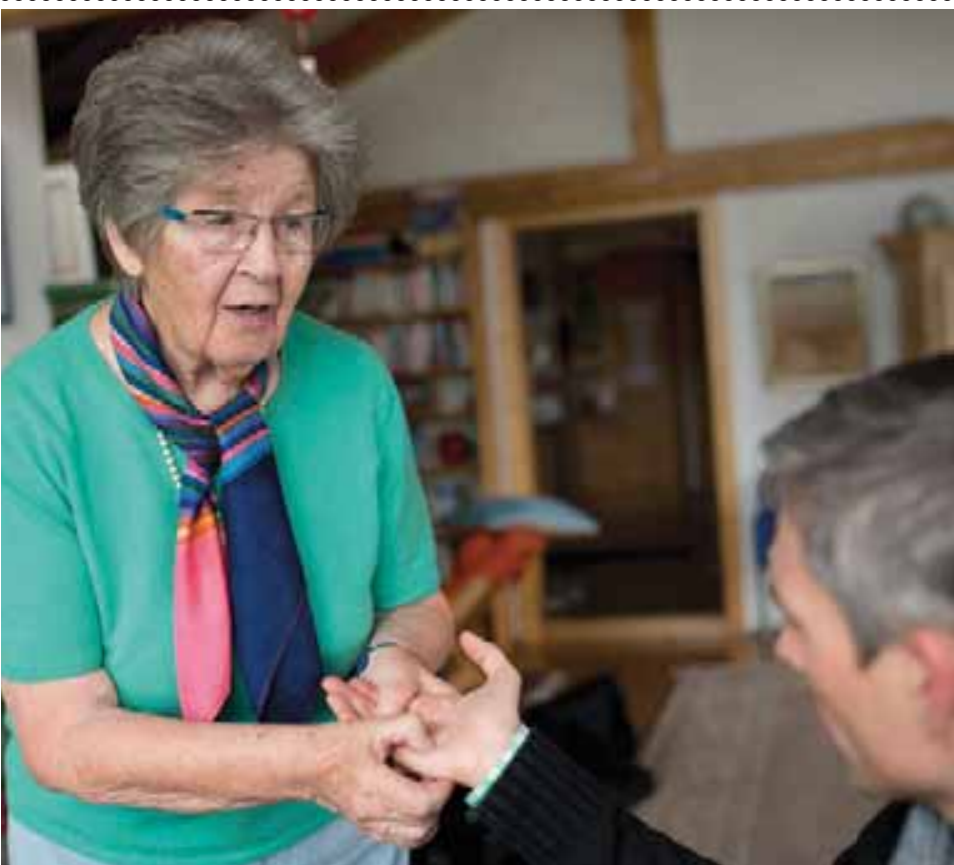

Alle Fotos: @ Oliver Lieber

sen hatte, lernte sie Ingham in einem ihrer Kurse in Toronto kennen. Die Amerikanerin lädt sie danach einige Tage zu sich in ihr Haus ein und bietet ihr eines Nachmittags eine Fußbehandlung an. „Damals gab es nur wenige Griffe, und die waren ziemlich hart. Passen Sie auf - so etwa." Hanne Marquardt lässt sich die linke Hand ihres Besuchers geben und drückt mit ihrem Daumen in das weiche Gewebe der Handinnenfläche zwischen dem Ringund kleinen Finger. Sie schiebt den Daumen nach oben und unten, drückt und kreist und drückt und kreist. „Nicht angenehm, oder?" Ja, das Gefühl ist sogar sehr unangenehm. „So etwa wurde vor mehr als 50 Jahren gearbeitet. Und mir wurde durch diese ,eindrückliche' Erfahrung bewusst, dass ich mit dem schmerzhaften Zonen an den Füßen meiner Patienten ab jetzt wesentlich sensibler umgehen würde." Monatelang probiert Hanne Marquardt daraufhin, bis ihr allmählich der Wechsel in der Technik gelingt: weg von mechanischem Druck hin zu dynamischer, rhythmischer Kraft. Auf dieser Basis entwickelt sie im Lauf der Jahre viele differenzierte Griffe, bis hin zu sanften Streichungen z. B. bei der Behandlung der Lymphzonen.

Der Besuch bei Eunice Ingham öffnet ihr aber noch für etwas ganz anderes die Augen. „Die Fußbehandlung führten in Amerika und vielen anderen Ländern damals hauptsächlich interessierte Laien durch, zur Prävention. " Für Hanne Marquardt war es von Beginn an aber selbstverständlich, therapeutisch zu arbeiten. So entscheidet sie sich 1967 nach ihrer Rückkehr aus Toronto, ihr Wissen an Therapeuten weiterzugeben, und beginnt neben der Praxis die Reflexzonentherapiezu unterrichten. 1975 schreibt sie ihr erstes Buch über die Behandlung an den Füßen, das mittlerweile in der 24. Auflage erschienen ist. In den frühen 1990er-Jahren dann ein weiteres, ausführlicheres Lehrbuch, inzwischen in der 7. Auflage erschienen.

\section{Und heute?}

Heute, über 40 Jahre danach, nehmen Heilpraktiker, Physiotherapeuten, Pflegende, Hebammen, Masseure, und auch Ärzte, an den Kursen zur Reflexzonentherapie am Fuß teil. Hanne-MarquardtFussreflex ${ }^{\circledR}$ heißt sie inzwischen und ist ein eingetragenes Mar- kenzeichen. Allein in Deutschland wird die Methode an acht, im europäischen und außereuropäischen Ausland an etlichen weiteren Ausbildungszentren nach einem von ihr entwickelten Qualitätsstandard unterrichtet.

Hanne Marquardt unterrichtet teilweise auch heute noch selber. Am Nachmittag dieses grauen Donnerstags im November steht sie vor einer Gruppe von zwölf Teilnehmern, erster Tag der Ausbildung in der Behandlung an den Füßen, und sie zeigt auf einem kleinen Bild die offensichtliche Ähnlichkeit zwischen einem Fuß und dem sitzenden Menschen. „Die Einfachheit dieser Abbildung ist für viele, die sich zum ersten Mal dem Thema stellen, fast eine Zumutung. Ich sehe manchen Gesichtern an, dass Sie genau das denken, was mir vor 56 Jahren durch den Kopf ging: So einfach kann es doch nicht sein! Aber wenn Sie dann an den Behandlungsbänken sitzen, mit den Füßen Ihrer Kolleginnen und Kollegen vor sich, dann sehen Sie: Doch, es kann so einfach sein." Dann bittet Hanne Marquardt eine der Kursteilnehmerinnen auf einer Behandlungsbänke Platz zu nehmen und zeigt der Gruppe die ersten Grifftechniken

Internet: www.fussreflex.de

Dieser Artikel ist online zu finden:

http://dx.doi.org/10.1055/s-0034-1371501 